\title{
Value of tube combined with real-time ultrasound-guided accurate interstitial high-dose-rate brachytherapy for post-operative pelvic side-wall recurrences of cervical cancer
}

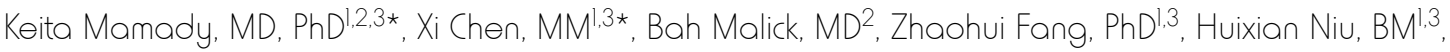 \\ Traoré Bangaly, BM², Hong Liu, PhD',3 \\ 'Department of Gynecologic Radiotherapy, The Fourth Affiliated Hospital of Hebei Medical University, Shijiazhuang, China, ${ }^{2 S}$ Surgical \\ Oncology Unit of Donka University Hospital, Conakry, Guinea, ${ }^{3}$ Hebei Provincial Key Laboratory of Tumor Microenvironment and Drug \\ Resistance, Shijiazhuang, China \\ *Keita Mamady and Xi Chen are contributed equally to this work.
}

\begin{abstract}
Purpose: Treatment of post-operative pelvic side-wall relapses of cervical cancer has always been a therapeutic challenge for radiation oncologists. Radiation dose boost to recurrent tumor by brachytherapy is necessary, but difficult to achieve. Our treatment center has successfully achieved precise transvaginal insertion of a pelvic side-wall mass, using a metal tube with real-time ultrasound guidance. This study investigates the efficacy and safety of image-guided high-dose-rate (HDR) interstitial brachytherapy (IBT) for patients with post-operative pelvic side-wall relapses.

Material and methods: Between 2018 and 2020, 36 post-operative pelvic side-wall relapses of cervical cancer patients receiving external beam radiotherapy (EBRT) combined with HDR-IBT were analyzed retrospectively. Doses per fraction ranged from 6.0 to $7.0 \mathrm{~Gy}$, whereas cumulative equivalent doses in 2 Gy fractions ranged from 80 to 100 Gy. Effects of prognostic factors on local control (LC) and progression-free survival (PFS) were analyzed, and late toxicity data were evaluated.

Results: A total of thirty-six patients were included, with a median follow-up of 19.3 months. The tumor response was obtained for all patients, with radiological complete remission in 20 (55.6\%) patients. Two-years LC and PFS rates were $72.2 \%$ and $47.2 \%$, respectively. Grade II rectal toxicity was observed in 5 patients (13.9\%). Multivariate analyses for LC and PFS using proportional regression model were performed, in which shape of exophytic tumor was associated with a significantly better prognosis for both LC and PFS (HR $=0.071,0.128, p=0.005,0.002)$. Clinical target volume (CTV) $\mathrm{D}_{90}$ remained associated with a significantly better prognosis for PFS (HR $\left.=0.056, p=0.000\right)$.

Conclusions: A metal tube placed in the vagina, under the guidance of real-time ultrasound in transvaginal IBT for pelvis masses can be accurately achieved. The shape of exophytic tumor and CTV $\mathrm{D}_{90}$ were associated with a significantly better prognosis for PFS, and the shape of exophytic tumor was also associated with a better prognosis for LC. Therefore, radiation dose boost using IBT can improve the prognosis of patients with post-operative pelvic side-wall
\end{abstract} recurrences of cervical cancer.

Key words: cervical cancer, relapse, external beam radiotherapy, interstitial brachytherapy.

\section{Purpose}

Treatment of patients with post-operative pelvic sidewall relapses of cervical cancer has always been a therapeutic challenge for radiation oncologists to deliver optimal irradiation doses, and to achieve acceptable clinical outcomes with minimal toxicities [1, 2]. Because of critical radio-sensitive organs, such as the small bowel, even with intensity-modulated radiation therapy (IMRT), it is difficult to deliver doses over $60 \mathrm{~Gy}$, while respecting normal tissue tolerance [3]. Usually, 60 Gy of radiation dose is not enough to control recurrent tumor on the pelvic side-wall [4]. In this setting, selective radiation dose boost to the tumor with brachytherapy is necessary to achieve better local control (LC) [5, 6]. Previously, interstitial brachytherapy (IBT) was often used for treatment of a relapsed disease in pelvic cancer [7,8]. For recurrent 
lesions in the pelvic side-wall, it was difficult to achieve accurate treatment through IBT, and therapeutic effect was not satisfactory. The main reason to be considered in IBT regarding a relapse in the pelvic side-wall is that the tumor cannot be accessed through the vagina. IBT operation through the perineum using free-hand technique is difficult and inaccurate, and real-time guidance of ultrasound is impossible. Up till now, not much literature is available for adequate IBT in selected group of patients with pelvic side-wall recurrences.

In the current study, we present our experience of using a metal tube combined with real-time ultrasound-guided accurate IBT after external beam radiotherapy (EBRT) in post-operative pelvic side-wall recurrences of cervical cancer. The aim of reporting this experience was to explain that 1 . If a metal tube is used to assist the implantation of recurrent pelvic masses at various parts, the process can be easily achieved under the guidance of real-time ultrasound; 2 . Through accurate IBT, a higher dose of radiation boost to the recurrent tumor can be accomplished, and some of these patients are expected to be cured; and 3. Clinical toxicities of the rectum, bladder, and bowel can be reduced in these selected patients of post-operative pelvic side-wall recurrences of cervical cancer.

\section{Material and methods}

\section{Patient selection}

Between February 2018 and May 2020, a total of 36 consecutive patients with post-operative pelvic side-

Table 1. Baseline patients' characteristics $(n=36)$

\begin{tabular}{lc}
\hline Parameter & Number \\
\hline Age (years) & \\
\hline Median (range) & $48(24-72)$ \\
\hline Pathological type & 31 \\
\hline Squamous carcinoma & 5 \\
\hline Adenocarcinoma & \\
\hline FIGO stage at initial diagnosis & 29 \\
\hline IA & 16 \\
\hline IB1 & 9 \\
\hline IB2 & 7 \\
\hline II & 28 \\
\hline Maximum tumor diameter $(\mathrm{cm})$ & 8 \\
\hline$<4$ & 30 \\
\hline$\geq 4$ & 6 \\
\hline Shape of tumor & 29 \\
\hline Exophytic shape & 7 \\
\hline Infiltrative shape & \\
\hline Tumor number & \\
\hline Single & \\
\hline Multiple & \\
\hline
\end{tabular}

FIGO - International Federation of Gynecology and Obstetrics wall recurrences of cervical cancer treated using IBT with EBRT were evaluated. Patients were regarded eligible according to the following inclusion criteria: 1 . Histological diagnosis of cervical cancer; 2 . Recurrent tumor located in the pelvic side-wall; 3 . No prior radiotherapy for cervical cancer; and 4. Written informed consent obtained from patient. Patients were excluded if any of the following exclusion criteria were fulfilled: 1 . Evidence of distant metastasis at the time of pelvic side-wall relapse diagnosis; 2. Patients with severe thrombocytopenia (platelet count $\leq 50 \times 10^{9} / 1$ ), which could not tolerate radiotherapy; 3. Contraindications for irradiation, including complete obstruction of the vagina and fistula in the vagina.

\section{Pre-treatment evaluation and clinical characteristics}

Pre-treatment evaluation consisted of history and physical examination. Extent of disease was evaluated based on computed tomography (CT), magnetic resonance imaging (MRI), and positron emission tomography/CT (PET/CT, if available). Patients' characteristics are shown in Table 1 . Median age was 48 years (range, 24 to 72 years). All patients presented histologically confirmed squamous cell carcinoma, except five (13.9\%) patients who had adenosquamous carcinoma. According to the International Federation of Gynecology and Obstetrics stages, at initial diagnosis, twenty-nine patients presented with stage I disease, and 7 were stage II. Twenty-eight $(77.8 \%)$ patients had a tumor with a clinical size $<4 \mathrm{~cm}$, and eight $(22.2 \%)$ had a clinical size $\geq 4 \mathrm{~cm}$. Baseline tumors' characteristics are demonstrated in Table 1. Almost all patients underwent EBRT and IBT. Whole pelvic EBRT was delivered by IMRT or image-guided radiotherapy (IGRT) for all patients. Clinical target volume (CTV) for EBRT included recurrent tumor, vaginal cuff, internal iliac, external iliac, common iliac, obturator, presacral lymph node, and para-aortic lymph node regions in cases of positive para-aortic lymph nodes [9]. CTV upper limit was half of the fourth lumbar vertebrae, and CTV lower limit included lower edge of the obturator. Planning target volume (PTV) was defined as a $10-\mathrm{mm}$ expansion around CTV. GTV corresponded to recurrent tumors in the pelvic cavity. Prescribed dose was 45 Gy to PTV, and 60 Gy to GTV in 25 fractions at a single-dose of 1.8-2.4 Gy per fraction. Dose-volume constraints were defined for the small bowel $\left(\mathrm{V}_{30}<40 \%, \mathrm{D}_{\max }<52 \mathrm{~Gy}\right)$, bladder $\left(\mathrm{V}_{45}<45 \%\right)$, and rectum $\left(\mathrm{V}_{45}<60 \%\right)$, which were adjacent to CTV. Concurrent chemotherapy with TP (paclitaxel $135 \mathrm{mg} / \mathrm{m}^{2}+$ cisplatin $70 \mathrm{mg} / \mathrm{m}^{2}$ ) regimen was given every three weeks to patients during and after radiotherapy, to a maximum of six cycles [10]. All patients were hospitalized and monitored weekly in the course of treatment for acute toxicity and symptomatic management.

\section{IBT implant procedure}

In our institution, brachytherapy is a component of definitive radiotherapy or concurrent chemo-radiotherapy for cervical cancer. Usually, this procedure is performed after the completion of EBRT [11]. A day before 
implantation procedure, patients are given an appropriate bowel preparation. A CT-scan with bladder filling $(100 \mathrm{ml})$ protocol to minimize internal organ motion was obtained before IBT and during the last week of EBRT, to assess treatment response. When post-EBRT response evaluation shows shrinking of the tumor of more than $50 \%$, it means higher sensitivity to radiotherapy, but if the tumor does not shrink or shrinks to $50 \%$, a higher dose of radiotherapy during IBT treatment is administered to the local tumor to achieve better tumor control. Therefore, during the implantation process, the tube was placed into the vagina without using a vaginal speculum. Under ultrasound guidance, the direction of the tube was adjusted until the tangent of longitudinal axis of the tube could directly reach the angle of the pelvic mass, without any anesthesia (Figure 1). We used a stainless steel metal tube with openings at both ends, $17 \mathrm{~cm}$ long and $1.5 \mathrm{~mm}$ in diameter. The longest insertion needle used is $20 \mathrm{~cm}$.
Interpolating needle was inserted directly into the tumor tissue along the tube. CT-scan (with 3-mm slice thickness) was performed to verify interstitial needle positioning, rectum, bladder volume, and small bowel distribution around the treatment site. If these were satisfactory, a radiation oncologist used these CT images to contour the target and organs at risk (OARs). The overall duration of EBRT plus IBT was aimed to be less than 57 days.

\section{Target delineation and treatment planning}

Brachytherapy inverse planning simulated annealing (IPSA) was performed using Oncentra Brachy v. 4.5.3. Target volume consisted of high-risk clinical target volume (HR-CTV) and OARs, including the rectum, bladder, and small bowel (Figure 2). HR-CTV was contoured according to GYN GEC-ESTRO recommendations [11, 12]. Doses to the bladder, rectum, and small bowel were de-
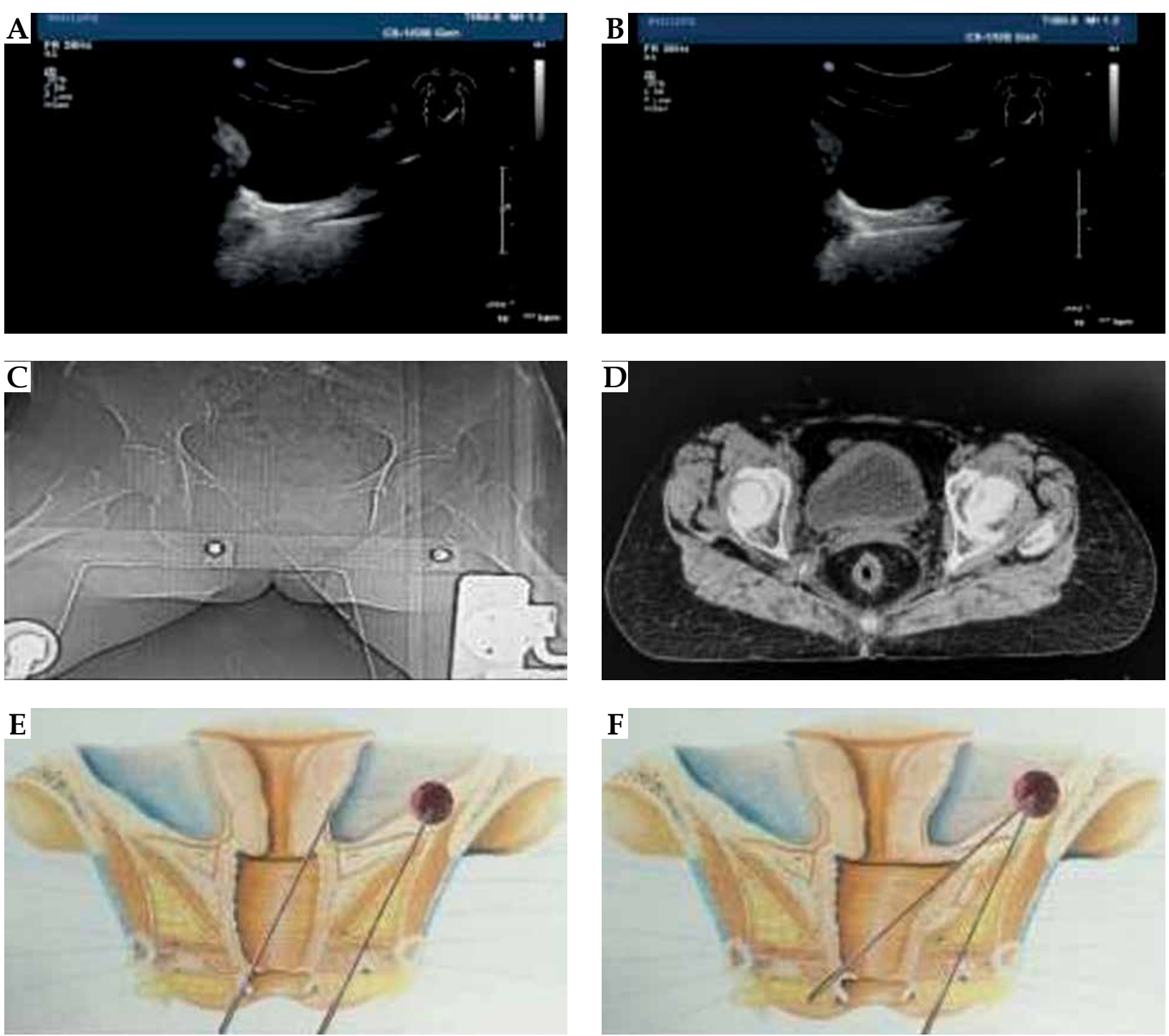

Fig. 1. A) Ultrasound shows the direction of metal tube in the vagina; B) In real-time guidance, the interstitial needle is inserted directly into the tumor tissue of the pelvic side-wall; C) Inserted needle is displayed in CT image; D) Inserted needle is displayed in CT across image; E) This illustration shows the maximum tilt angle of transvaginal needle using vaginal speculum; F) This illustration shows the maximum tilt angle of transvaginal needle without the use of vaginal speculum 


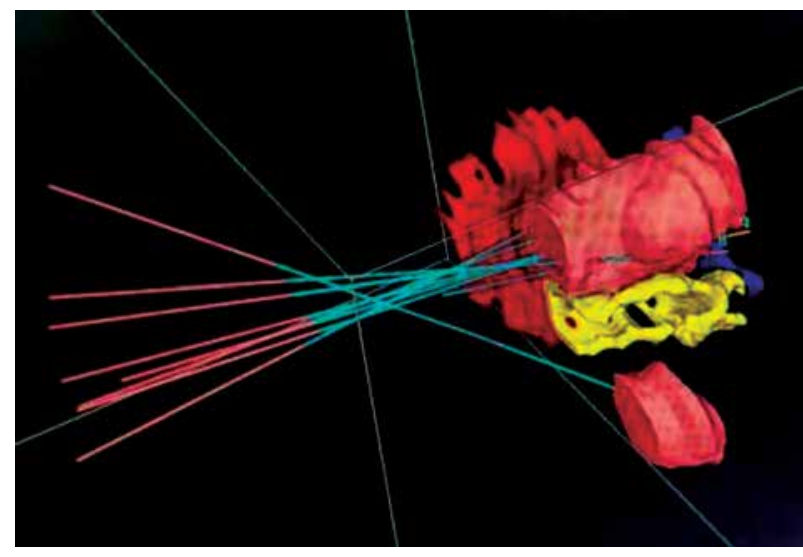

Fig. 2. Three-dimensional reconstruction of the contoured volumes, showing bladder (red), rectum (yellow), and clinical target volume (pink) in the tumor region

termined according to the International Commission on Radiation Units (ICRU) requirements [13]. Appropriate dose prescription was assigned for all OARs. At the same time, $\mathrm{D}_{2 \mathrm{cc}}$ volume of the rectum and bladder should not receive more than $60 \%$ and $80 \%$ of the prescribed dose, respectively. Biologically effective dose (BED) was calculated for HR-CTV using the equation for radio-biological equivalence, with standard conventional fractionation suggested by Fowler, as follows: BEDfr $=\mathrm{ND}\{1+\mathrm{d}$ / $(\alpha / \beta)\}$, where $N$ is radiotherapy treatment fractions' number, $d$ is dose per fraction in Gy, and $\alpha / \beta$ is tissue-specific parameter depending on whether it is early or late reaction to radiotherapy. The value of $\alpha / \beta$ was taken as 10 for tumor and early reacting tissues, such as the bladder, rectum, and small bowel, and 3 for late reacting tissues, such as muscle and nerve [14,15]. Cumulative doses of EBRT

Table 2. Treatment characteristics of interstitial brachytherapy

\begin{tabular}{lc} 
Parameter & Number \\
\hline No. interstitial needles & 6 \\
\hline Mean & $1-14$ \\
\hline Range & 10.9 \\
\hline IBT total dose (Gy) & $9-12$ \\
\hline Mean & 14.7 \\
\hline Min.-Max. & $11.7-16.8$ \\
\hline IBT BED dose (Gy) & $79.6 \pm 0.2$ \\
\hline Mean & $62.1 \pm 0.4$ \\
\hline Min.-Max. & $94.5 \%(435 / 460)$ \\
\hline Bladder D $D_{2 c c}(G y)$ & 0.0 \\
\hline Rectum $D_{2 c c}(G y)$ & 0.0 \\
\hline Effective needles inserted & 0.0
\end{tabular}

IBT - interstitial brachytherapy, BED - biological effective dose, Min. - minimum, Max. - maximum and IBT to the bladder, rectum, and the small bowel were calculated to achieve optimal coverage, where HR-CTV receives optimum dose without affecting the tolerances of these critical OARs. After approval of the treatment plan, the patient was taken to brachytherapy room for the treatment. Implant needles could be removed immediately after the completion of the treatment.

\section{Post-treatment patient follow-up}

Patients were followed-up every three months, up to two years. At each visit, a clinical examination was performed to evaluate treatment response, and to assess any treatment-related complications. During the treatment, hematological toxicity was assessed for each patient using the World Health Organization (WHO) common acute and sub-acute toxicity criteria for anti-cancer drugs [16]. CT of the whole abdomen and pelvis was performed at third and sixth months for accurate assessment of local response.

\section{Statistical analysis}

All of the statistical analyses were performed using SPSS version 23.0 software (SPSS Inc., Chicago, IL, USA). Local control was determined as the time from diagnosis of recurrence to progression of disease in the pelvic cavity. Progression-free survival (PFS) was calculated from the diagnosis of recurrence to the progression of target lesions or the occurrence of new lesions. Log-rank tests were used to compare LC and PFS rates. The parameters analyzed were LC and PFS using univariate and multivariate Cox regression analysis, considering age at recurrence, pathological type, maximum tumor diameter, hemoglobin level, time to surgery, chemotherapy, shape of the tumor, number of tumors, and CTV $\mathrm{D}_{90}$. The level of significance was set as $p<0.05$.

\section{Results}

All the patients received EBRT combined with IBT. The EBRT duration ranged from 35 to 40 days. When post-EBRT response revealed more than $50 \%$ of disease volume regression, a partial response was considered. All patients received concurrent chemotherapy with curative intent, except for five patients who experienced non-tolerance or other medical comorbidities. The treatment characteristics are shown in Table 2. One out of fourteen needles were placed per fraction, with a mean of 6 needles. The planning CT scan did not reveal any organ penetration due to needle placement. Dose to target volumes and OAR structures were summed up, and $\mathrm{EQD}_{2}$ was calculated. The dose to HR-CTV ranged from 18 to $28 \mathrm{~Gy}$ (median, 24.3 Gy). Conversion to equivalent dose in $2 \mathrm{~Gy} / \mathrm{fx}$. $\left(\mathrm{EQD}_{2}\right)$ to the target volume ranged from 24.0 to $39.67 \mathrm{~Gy}$. The doses received by $90 \%\left(\mathrm{D}_{90}\right)$ and $100 \%\left(\mathrm{D}_{100}\right)$ of the HR-CTV conversion to $\mathrm{EQD}_{2}$ ranged from 24.0 to $39.67 \mathrm{~Gy}$ and 20.47 to $28.93 \mathrm{~Gy}$, respectively. The mean cumulative $2 \mathrm{cc}$ rectal and bladder doses were $62.1 \pm 0.4 \mathrm{~Gy}$ and $79.6 \pm 0.2 \mathrm{~Gy}$, respectively (including the doses received by EBRT). Dose limits for total $\mathrm{EQD}_{2}$ of OARs were $<90 \mathrm{~Gy}$ for the bladder, $<75 \mathrm{~Gy}$ for the rectum, 
Table 3. Patients' toxicities after irradiation

\begin{tabular}{|c|c|c|c|c|c|c|c|c|c|c|}
\hline \multirow[t]{2}{*}{ Toxicity } & \multicolumn{2}{|c|}{ Grade 0} & \multicolumn{2}{|c|}{ Grade 1} & \multicolumn{2}{|c|}{ Grade 2} & \multicolumn{2}{|c|}{ Grade 3} & \multicolumn{2}{|c|}{ Grade 4} \\
\hline & $\begin{array}{c}\text { No. } \\
\text { of patients }\end{array}$ & $\%$ & $\begin{array}{c}\text { No. } \\
\text { of patients }\end{array}$ & $\%$ & $\begin{array}{c}\text { No. } \\
\text { of patients }\end{array}$ & $\%$ & $\begin{array}{c}\text { No. } \\
\text { of patients }\end{array}$ & $\%$ & $\begin{array}{c}\text { No. } \\
\text { of patients }\end{array}$ & $\%$ \\
\hline Gl reaction & 0 & 0.0 & 23 & 63.8 & 13 & 36.1 & 0 & 0.0 & 0 & 0.0 \\
\hline Radiation enteritis & 3 & 8.3 & 19 & 52.8 & 14 & 38.9 & 0 & 0.0 & 0 & 0.0 \\
\hline Radiation cystitis & 12 & 33.3 & 11 & 30.6 & 3 & 8.3 & 0 & 0.0 & 0 & 0.0 \\
\hline Myelosuppression & 5 & 13.9 & 9 & 25.0 & 22 & 61.1 & 0 & 0.0 & 0 & 0.0 \\
\hline
\end{tabular}

Gl reaction - gastrointestinal reaction

$<75$ Gy for the sigmoid colon, and $<75$ Gy for the small bowel. Radiotherapy-associated toxicities were evaluated with common terminology criteria for adverse events (CTCAE), version 4.0 (Table 3) [17]. No treatment-related grade 3 toxicity was reported. Grade 2 rectal toxicity was seen in five patients, and the median $\mathrm{D}_{2 \mathrm{cc}}$ rectal dose was 78.8 Gy. Radiation proctitis occurred during 6 to 18 months after completion of treatment, with a median of 10 months. A strong correlation with dose delivered to the rectum was noted. The largest HR-CTV volume involved in the present study was $148 \mathrm{~cm}^{3}$ and the smallest was
$3.77 \mathrm{~cm}^{3}$. The average volume of HR-CTV was $41.24 \mathrm{~cm}^{3}$. Radiation proctitis was also correlated with large HRCTV volumes $(>130 \mathrm{ml})$ and those approaching close to the rectal mucosa. Dose distribution to the whole bowel was insignificant due to high conformity, even though the bowel was close to target volume. However, after one year, two patients showed complications, including numbness, pain, and even limping claudication of the affected side. Tumor response after IBT was obtained in all the patients, and complete remission (CR) was confirmed radiologically in 20 cases. Disease progression during fol-

Table 4. Univariate analysis of local control (LC) and progression-free survival (PFS) in 36 patients with recurrent cervical cancer

\begin{tabular}{|c|c|c|c|c|}
\hline Prognosis factors & 2-year LC rate (\%) & $p$-value & 2-year PFS rate (\%) & $p$-value \\
\hline Age at irradiation & & 0.782 & & 0.571 \\
\hline$<50$ years & 79.2 & & 50.0 & \\
\hline$\geq 50$ years & 83.3 & & 58.3 & \\
\hline Pathological type & & 0.351 & & $0.036^{*}$ \\
\hline Squamous carcinoma & 83.3 & & 63.3 & \\
\hline Adenocarcinoma & 66.7 & & 16.7 & \\
\hline Maximum tumor diameter & & $0.036^{*}$ & & $0.003^{*}$ \\
\hline$<4 \mathrm{~cm}$ & 91.3 & & 73.9 & \\
\hline$\geq 4 \mathrm{~cm}$ & 61.5 & & 23.1 & \\
\hline Hemoglobin level & & $0.002^{*}$ & & $0.013^{*}$ \\
\hline$<8.0 \mathrm{~g} / \mathrm{dl}$ & 33.3 & & 16.7 & \\
\hline$\geq 8.0 \mathrm{~g} / \mathrm{dl}$ & 90.0 & & 63.3 & \\
\hline Interval to operation & & 0.611 & & 0.986 \\
\hline$<12$ months & 77.3 & & 54.4 & \\
\hline$\geq 12$ months & 85.7 & & 57.1 & \\
\hline Chemotherapy & & 0.468 & & 0.833 \\
\hline Yes & 81.8 & & 66.7 & \\
\hline No & 66.7 & & 54.5 & \\
\hline Shape of tumor & & $0.000^{*}$ & & $0.000^{*}$ \\
\hline Exophytic shape & 93.3 & & 66.7 & \\
\hline Infiltrative shape & 16.7 & & 0.0 & \\
\hline Tumor number & & $0.003^{*}$ & & $0.001^{*}$ \\
\hline Single & 89.7 & & 66.7 & \\
\hline Multiple & 42.9 & & 0 & \\
\hline CTV $D_{90}$ & & $0.003^{*}$ & & $0.000^{*}$ \\
\hline$<85$ Gy & 57.1 & & 7.1 & \\
\hline$\geq 85$ Gy & 95.1 & & 86.4 & \\
\hline
\end{tabular}


Table 5. Multivariate analysis of survival outcomes in 36 patients with recurrent cervical cancer

\begin{tabular}{lcccccc} 
& \multicolumn{3}{c}{ Local control } & \multicolumn{3}{c}{ Progression-free survival } \\
\cline { 2 - 7 } & $\operatorname{Exp}(\mathrm{B})$ & $95 \% \mathrm{Cl}$ & $p$-value & $\operatorname{Exp}(\mathrm{B})$ & $95 \% \mathrm{Cl}$ & $p$-value \\
\hline CTV D $_{90}$ & 0.109 & $0.012-1.016$ & 0.052 & 0.056 & $2.141-28.550$ & $0.000^{*}$ \\
\hline Shape of tumor & 0.071 & $2.199-89.299$ & $0.005^{*}$ & 0.128 & $0.015-0.213$ & $0.002^{*}$
\end{tabular}

LC - local control; PFS - progression-free survival

low-up was detected in 16 patients, with 7 patients experiencing a local recurrence, and 9 patients suffering from a distant metastatic disease. The LC rate at two years was $80.9 \%$. The univariate analyses of LC and PFS with logrank test were performed according to age, pathological type, maximum tumor diameter, hemoglobin level, interval time to operation, chemotherapy, tumor shape, tumor number, and CTV $\mathrm{D}_{90}$ (Table 4). Among all these factors, maximum tumor diameter < $4 \mathrm{~cm}$ (2-year LC: $91.3 \%$ vs. $61.5 \%, p=0.036$ ), hemoglobin level $\geq 8.0 \mathrm{~g} / \mathrm{dl}$ (2-year LC: $90.0 \%$ vs. $33.3 \%, p=0.002$ ), exophytic shape (2-year LC: $93.3 \%$ vs. $16.7 \%, p=0.000$ ), single-tumor (2-year LC: $89.7 \%$ vs. $42.9 \%, p=0.003$ ), and CTV $D_{90} \geq 85$ Gy (2-year LC: $95.5 \%$ vs. $57.1 \%, p=0.003$ ) were associated with a significantly better prognosis for LC. In addition to pathological type squamous carcinoma (2-year PFS: 63.3\% vs. $16.7 \%, p=0.036)$ and hemoglobin level $\geq 8.0 \mathrm{~g} / \mathrm{dl}$ (2-year PFS: $16.7 \%$ vs. $63.3 \%, p=0.013$ ), maximum tumor diameter < $4 \mathrm{~cm}$ (2-year PFS: $73.9 \%$ vs. $23.1 \%$, $p=0.003$ ), exophytic shape (2-year PFS: $66.7 \%$ vs. $0.0 \%$, $p=0.000$ ), single-tumor (2-year PFS: $65.5 \%$ vs. $14.3 \%$, $p=0.001)$, CTV $D_{90} \geq 85$ Gy (2-year PFS: $86.4 \%$ vs. $7.1 \%$, $p=0.000$ ) were all found to be significantly associated with a better prognosis for PFS (Table 4). Multivariate analyses for LC and PFS using proportional regression model were performed, with the shape of tumor and CTV $\mathrm{D}_{90}$ remaining associated with a significantly better prognosis for PFS $(p<0.05)$. Moreover, the shape of tumor was the only factor associated with a better prognosis for LC $(p<0.05)$ (Table 5).

\section{Discussion}

Cervical cancer patients with vaginal vault recurrences or residual diseases after surgery, treated with conventional radiotherapy have been associated with five years locoregional control rates of $20-60 \%$ [18]. Patients with pelvic side-wall recurrences after surgery for cervical cancer, who were treated with EBRT alone have rarely been reported cured. Treatment of residual and recurrent cervical cancer of pelvic side-wall recurrence poses a therapeutic challenge to radiation oncologists, because of various limitations. The main limitations concern operating technique and constraints related to OARs $[19,20]$. It is difficult to deliver higher doses to target volume with EBRT beyond $60 \mathrm{~Gy}$, even if IMRT/IGRT techniques are used because after surgery, the small bowel falls into the true pelvis and approaches close to the target area. Therefore, it is impossible to treat such patients using EBRT only, and at the same time to essentially reduce the dose to the small bowel, bladder, and rectum. Higher doses of radiation could be delivered to the pelvic side-wall tumor using IBT after EBRT, but the principle is that interstitial needles can be inserted precisely into the tumor tissue. However, it is common that IBT needles cannot reach up to these pelvic side-wall lesions, therefore, cannot deliver an adequate dose [21].

Although IBT is widely available, the majority of published data on this technique is only for relapse tumors in the central pelvic. There has been no reported data on IBT treatment for pelvic side-wall recurrences. The reason is transvaginal IBT of the pelvic side-wall mass that has long been considered impossible (Figure 1E). At present, IBT treatment for pelvic side-wall recurrence is mainly achieved by free-hand technique through trans-perineal insertion (Figure 1F). As we all know, perineal insertion by free-hand is difficult and positioning is not accurate. In particular, real-time guidance of ultrasound or CT cannot be realized. Therefore, the precision of insertion cannot be guaranteed during trans-perineal implantation.

Many authors showed that high quality use of brachytherapy was predicted to be a significant factor for overall survival in multivariate analysis for recurrent cervical cancer [21]. While we know the importance of high quality brachytherapy, the key issue is to know how to insert the interstitial needle conveniently and accurately. The current work presented our experience using a tube combined with real-time ultrasound-guided accurate interstitial high-dose-rate brachytherapy for re-treatment of locally recurrent tumors to boost the acceptance radiotherapy dose. This research found that transvaginal implantation of pelvic masses could be effectively solved by using only a metal tube guided by real-time ultrasound. As described in the methods section, without the use of a vaginal speculum, we can use the characteristics of vaginal malleability through a metal tube, which is guided by ultrasound to adjust the metal tube to an appropriate angle; then, we can accurately implement the needles into the tumor along the tube. This method can not only deliver transvaginal implantation of pelvic side-wall masses, but also be completed under real-time guidance of ultrasound, which improves the precision and safety of implantation. It is well-known that this is impossible to achieve under the condition of operation using a vaginal speculum. Through this technique, we have achieved precise implantation into masses located at the pelvic side-wall through the vagina. This procedure not only shortens operation time, but also reduces pain of the patient, and organs at risk can be tolerated. Real-time guidance of ultrasound achieves accurate and safe implantation, improves the suitability of target area, and delivers higher-dose radiotherapy for recurrent tumors. Multivariate statistical analysis showed that CTV $\mathrm{D}_{90}$ and shape of the tumor were closely related to patient's PFS, and shape of the tumor was associated with a better prognosis for LC. Shape of the tumor in clinical implantation treat- 
ment process directly affects effective distribution of the implantation needle. The exophytic shape of the tumor allows for better distribution of the inserted needles and achieving better dose distribution. In the current study, the radiation doses used for IBT varied from 6 to 7 Gy per session in 3-5 fractions, with different LC and complication rates. Of note, a higher radiotherapy dose for recurrent tumor next to the pelvic side-wall was applied, especially when the tumor was located near the obturator region. It was observed that two patients showed numbness, pain, and even limping claudication of the affected side. CT imaging of these two patients with claudication showed that the local lesions had completely regressed. Reasons for this complication were analyzed, such as being related to local radiotherapy dose that reached a cumulative total of $100 \mathrm{~Gy}$, and lesions were all located in the obturator hole adjacent to the pelvic wall. At first, the patient developed numbness in the lower limb of the affected side, about 1 year after the completion of radiotherapy, and then further developed pain in the same limb of the affected side. The symptoms of claudication occurred due to worsening of pain. Therefore, as the recurrent pelvic wall lesions in these two patients were well controlled, so in our analysis, this was caused by a radiation response for late reacting tissues, such as muscle and nerve. In this study, the late toxicity reactions observed in patients before the submission of the article were mainly grade 2 rectal reactions, which were characterized by intermittent mucus and blood in stools as well as increased frequency of stools. As some patients had a late onset of late toxicity due to radiotherapy, it is possible that some late toxicity may have been underestimated due to relatively short follow-up period of this study. At present, we have not yet found an appropriate dose that can not only control recurrent tumors, but also does not trigger a pyriform syndrome-like response. However, apart from IBT, robotic stereotactic radiotherapy can also offer a short and well-tolerated treatment for lateral pelvic recurrences tumors [23]. Regardless of the type of radiotherapy technique, one of the challenges of treating very lateralized pelvic recurrence is that better local control achieved by dose escalation has to be balanced with toxicities due to high radiation dose to lymphatics pathways and damage to neuromuscular bundle.

Overall, in our study, we found that patients with pelvic side-wall recurrence, whose local radiotherapy dose was boosted with IBT could achieve a good LC and survival rates. The advantages of this method of using a tube combined with real-time ultrasound-guided treatment are shown as follow. First, operation through the vagina into the needle path is relatively short; the patient's pain is less, without anesthesia. Second, real-time ultrasonic guidance can be performed to ensure accuracy of the interstitial needle. Moreover, local residual tumor received a higher radiotherapy dose, which significantly improved the prognosis of these patients. In patients with failed treatment, the recurrent lesions generally showed an invasive growth state before radiotherapy and low hemoglobin level. In addition, due to a large size and irregular shape of the tumor as well as proximity of organs at risk, insufficient dose of HR-CTV $\mathrm{D}_{90}$ and failure of the treatment occurred. In this study, we found that in some patients with large HR-CTV, in whom the tumor developed exogenously and recurrence position was in a way from the rectum and bladder, tolerable dose of organ at risk could be met if HR-CTV could be achieved. Also, CTV $\mathrm{D}_{90}$ dose would also be achieved, with good rate of control. This research suggests that EBRT supplemented with metal tube combined with accurate real-time ultrasound-guided IBT for post-operative pelvic side-wall recurrence of cervical cancer, appears to be a practical and appropriate approach to provide optimal therapeutic benefits, with fewest side-effects. The real-time ultrasonic guidance assisted by a metal tube may not only avoid loading the needles piercing the bowel, bladder, and rectum, but also it can deliver a high-dose to target volumes. This helped us to achieve a high local control rate, with acceptable treatment-related complications.

Long-term follow-up with a larger number of patients would be necessary to further assess the effective radiation dose on local control as well as the overall outcome of selected patients

\section{Acknowledgments}

We would like to thank our colleagues for providing their continuous support as well as patients for their cooperation.

\section{Financial support}

The authors are grateful to the Natural Science Foundation of Hebei Province (No., H2021206429).

\section{Compliance with ethical standards}

The research involved human participants only and institutional ethical clearance was obtained. Informed consent was obtained from every participant of the study in the presence of a neutral witnesses.

\section{Disclosure}

The authors report no conflict of interest.

\section{References}

1. Ager BJ, Torgeson A, Francis SR et al. Impact of brachytherapy boost and dose-escalated external beam radiotherapy in margin positive cervical cancer treated with chemotherapy and radiation. Am J Clin Oncol 2020; 43: 35-42.

2. Toita T, Kitagawa R, Hamano T et al. Phase II study of concurrent chemoradiotherapy with high-dose-rate intra cavitary brachytherapy in patients with locally advanced uterine cervical cancer: Efficacy and toxicity of a low cumulative radiation dose schedule. Gynecol Oncol 2012; 126: 211-216.

3. Okazaki S, Murata K, Noda SE et al. Dose-volume parameters and local tumor control in cervical cancer treated with central-shielding external-beam radiotherapy and CT-based image-guided brachytherapy. J Radiat Res 2019; 60: 490-500.

4. Nakata M, Yoshida K, Shimbo T et al. High-dose-rate interstitial brachytherapy with hypoxic radiosensitizer KORTUC II for unresectable pelvic sidewall recurrence of uterine cervical cancer: a case report. J Contemp Brachytherapy 2020; 12: 606-611. 
5. Chargari C, Renard S, Espenel S et al. Can stereotactic body radiotherapy replace brachytherapy for locally advanced cervical cancer? French society for radiation oncology statement. Cancer Radiother 2020; 24: 706-713.

6. Milickovic N, Tselis N, Karagiannis E et al. Iridium-knife: another knife in radiation oncology. Brachytherapy 2017; 16: 884e892.

7. Tiwari R, Narayanan GS, Narayanan S et al. Long-term effectiveness and safety of image-based, transperineal combined intracavitary and interstitial brachytherapy in treatment of locally advanced cervical cancer. Brachytherapy 2020; 19: 73-80.

8. Masui K, Yamazaki H, Suzuki G et al. Small bowel perforation caused by applicator implantation in high-dose-rate interstitial brachytherapy for recurrent pelvic tumor: a case report. J Contemp Brachytherapy 2020; 12: 188-192.

9. Cihoric N, Tapia C, Krüger K et al. IMRT with 18FDGPETyCT based simultaneous integrated boost for treatment of nodal positive cervical cancer. Radiat Oncol 2014; 9: 83.

10. Zhao H, Li L, Su H et al. Concurrent paclitaxel/cisplatin chemoradiotherapy with or without consolidation chemotherapy in high-risk early-stage cervical cancer patients following radical hysterectomy: preliminary results of a phase III randomized study. Oncotarget 2016; 7: 70969-70978.

11. Schernberg A, Bockel S, Annede P et al. Tumor shrinkage during chemoradiation in locally advanced cervical cancer patients: prognostic significance, and impact for image-guided adaptive brachytherapy. Int J Radiat Oncol Biol Phys 2018; 102: 362-372

12. Pötter R, Haie-Meder C, Van Limbergen E et al. Recommendations from gynecological (GYN) GEC ESTRO working group (II): Concepts and terms in 3D image-based treatment planning in cervix cancer brachytherapy-3D dose volume parameters and aspects of 3D image-based anatomy, radiation physics, radiobiology. Radiother Oncol 2006; 78: 67-77.

13. Jamema SV, Saju S, Mahantshetty U et al. Dosimetric evaluation of rectum and bladder using image-based CT planning and orthogonal radiographs with ICRU 38 recommendations in intracavitary brachytherapy. J Med Phys 2008; 33: 3-8.

14. Fowler JF. The linear quadratic formula and progress in fractionated radiotherapy: a review. Br J Radiol 1989; 62: 679-694.

15. Siebert FA, Born T, Haring S et al. A dosimetric analysis of interstitial intensity modulated implants for pelvic recurrences, base of tongue and orbital tumors with specific references to the ICRU-58. Radiother Oncol 2006; 79: 298-303.

16. Lheureux S, Clarisse B, Launay-Vacher V et al. Evaluation of current practice: management of chemotherapy-related toxicities. Anticancer Drugs 2011; 22: 919-925.

17. Kluetz PG, Chingos DT, Basch EM et al. Patient-reported outcomes in cancer clinical trials: measuring symptomatic adverse events with the national cancer institute's patient-reported outcomes version of the common terminology criteria for adverse events (PRO-CTCAE). Am Soc Clin Oncol Educ Book 2016; 35: 67-73.

18. Coia L, Won M, Lanciano R et al. The patterns of care outcome study for cancer of uterine cervix. Cancer 1990; 66: 2451-2456.

19. Zolciak-Siwinska A, Bijok M, Jonska-Gmyrek J et al. HDR brachytherapy for the reirradiation of cervical and vaginal cancer: Analysis of efficacy and dosage delivered to organs at risk. Gynecol Oncol 2014; 132: 93-97.

20. Mahantshetty U, Kalyani N, Engineer R et al. Reirradiation using high-dose-rate brachytherapy in recurrent carcinoma of uterine cervix. Brachytherapy 2014; 13: 548-553.

21. Jurado M, Alcazar JL, Martinez-Monge R. Resectability rates of previously irradiated recurrent cervical cancer (PIRCC) treated with pelvic exenteration: Is still the clinical involve- ment of the pelvis wall a real contraindication? A twenty-year experience. Gynecol Oncol 2010; 116: 38-43.

22. Bansal V, Kumar G, Bhutani R et al. Three-dimensional conformal brachytherapy boost in locally recurrent or residual cervical carcinoma: Does it impact clinical outcome? J Obstet Gynaecol Res 2013; 39: 264-271.

23. Dewas S, Bibault JE, Mirabel X et al. Robotic image-guided reirradiation of lateral pelvic recurrences: preliminary results. Radiat Oncol 2011; 6: 77. 